\title{
HISTEROSCOPIA DIAGNÓSTICA: RENDIMIENTO EN EL ESTUDIO DE LA METRORRAGIA E INOCUIDAD ANTE UN EVENTUAL CÁNCER DE ENDOMETRIO, REVISIÓN DE LA LITERATURA
}

\author{
Juan Pablo Goycoolea T.1,2, Iván Rojas T.1,2 \\ ${ }^{1}$ Unidad Oncología Ginecológica, Hospital Padre Hurtado. ²Servicio de Ginecología y Obstetricia, Clínica Santa María.
}

\section{RESUMEN}

Objetivo: Evaluar la precisión diagnóstica de la histeroscopia en el estudio del endometrio en pacientes con metrorragia y determinar los riesgos derivados de efectuar el examen en pacientes portadoras de un cáncer de endometrio. Método: Revisión no sistemática de la literatura basada en MEDLINE y rastreo de referencias de artículos relevantes. Resultados: Se identificaron 7 metaanálisis, los cuales incluyen 43.757 pacientes sometidas a diferentes procedimientos para el estudio del endometrio. Se compara el rendimiento de la histeroscopia diagnóstica contra biopsia aspirativa endometrial, ecografía transvaginal e histerosonografía en su correlación con la histología. La histeroscopia resultó ser un examen de muy alta especificidad al igual que la biopsia espirativa, que los hace útiles en el estudio de casos con endometrio anormal seleccionados por ecografía transvaginal. Se examinó las recomendaciones autorizadas, la evidencia a favor y en contra de realizar histeroscopia en pacientes con cáncer de endometrio. Conclusiones: No existe evidencia suficiente para sostener la inocuidad de la histeroscopia diagnóstica en este tipo de pacientes, pero tampoco para afirmar que empeore su pronóstico. Se propone un esquema de estudio de la metrorragia en diferentes situaciones clínicas en base a la evidencia disponible, teniendo en cuenta la sugerencia de evitar en lo posible la histeroscopia en pacientes con riesgo alto de cáncer de endometrio.

\section{PALABRAS CLAVES: Histeroscopia, metaanálisis, cáncer endometrial, sangrado uterino anormal, metrorragia postmenopausia}

\section{SUMMARY}

Objective: To asses the accuracy of diagnostic hysteroscopy in the study of endometrium in patients with abnormal bleeding and to determine the risks of this procedure in patients with endometrial cancer. Methods: Non systematic review of MEDLINE based literature and manual search of relevant articles references. Results: Seven meta-analysis were identified. They included 43,757 patients who underwent through different endometrial diagnostic tests. In this data, diagnostic accuracy of hysteroscopy was compared against endometrial sampling, transvaginal ultrasound and hysterosonography. The gold standard was the anatomo pathologic findings. Hysteroscopy and endometrial sampling were found to have the highest specificitiy, which make them useful tests in the management of women with abnormal endometrial thickness in transvaginal ultrasound. Authorized recommendations were reviewed as well as the evidence in favor and against of performing hysteroscopy in endometrial cancer patients. Conclusion: There is neither enough evidence to support the safety of diagnostic hysteroscopy in this group of patients, nor to state that the procedure correlates with worse prognosis. Based on the existing evidence, the authors propose a study 
protocol of abnormal uterine bleeding in different clinical settings, considering the general advice to avoid hysteroscopy as possible in high risk patients for endometrial cancer.

\section{KEY WORDS: Hysteroscopy, meta-analysis, endometrial cancer, abnormal uterine bleeding, postmenopausal bleeding}

\section{INTRODUCCIÓN}

El sangrado uterino anormal, principalmente la metrorragia, es el síntoma más frecuente de la patología endometrial y constituye una consulta ginecológica de alta prevalencia en premenopáusicas $(1,2)$. Entre sus causas están: sangrado disfuncional secundario a anovulación, miomas submucosos, pólipos endometriales e hiperplasia endometrial. Menos frecuentes en general son el cáncer endometrial y otros tumores malignos, los cuales son excepcionales en mujeres jóvenes. Es necesario estudiar 3.000-4.000 mujeres con alteraciones de flujos rojos menores de 40 años para encontrar un cáncer endometrial (3). En contraste, alrededor del $10 \%$ de las metrorragias postmenopáusicas presenta un cáncer uterino, principalmente de endometrio, existiendo series que oscilan entre $5-60 \%$ de los casos, dependiendo de los factores de riesgo presentes (4).

Los exámenes tradicionalmente utilizados para el estudio de la metrorragia son ultrasonografía transvaginal (EcoTv), histerosonografía (HSG), histeroscopia $(\mathrm{Hx})$, biopsia aspirativa endometrial (BEM) y legrado formal. Los criterios para definir qué estrategia de estudio y qué tipo de exámenes usar en diferentes situaciones clínicas no están claramente establecidos. Ello ocasiona que existan opiniones diversas sobre el rendimiento y seguridad de ciertos exámenes y en particular de la histeroscopia.

El objetivo de la presente revisión es responder a 3 preguntas clínicas relevantes:

1. La histeroscopia, ¿tiene riesgos o es inocua en el manejo de una paciente portadora de un cáncer endometrial?

2. ¿Cuál es el rendimiento diagnóstico de la histeroscopia en una paciente con sangrado anormal?

3. En base a la evidencia disponible, ¿es posible definir un esquema de estudio de la metrorragia para diferentes situaciones clínicas?

\section{EFECTO DE LA HISTEROSCOPIA SOBRE EL CÁNCER DE ENDOMETRIO}

En el congreso anual de la Sociedad Norteamericana de Ginecólogos Oncólogos (SGO) del año 2000, son dadas a conocer (5) las investigaciones de Zerbe y cols (6) y de Sonoda y cols (7) que alertan sobre una posible iatrogenia relacionada con la manipulación uterina de pacientes con cáncer endometrial. Ambos trabajos mostraron diferencias significativas en términos de un mayor porcentaje de citologías positivas para células neoplásicas en cavidad abdominal posterior a histeroscopia prehisterectomía: $17 \%$ vs. $6,3 \%$ (6); y en relación al uso de manipulador uterino durante la histerectomía: 13 vs. $3,6 \%$ (7).

La posición de la SGO en relación a la manipulación uterina de pacientes con cáncer endometrial y al posible aumento de estadios IIIA asociado a estas maniobras, quedó establecida entonces (5) en las siguientes aseveraciones:

1. El estadio IIIA del cáncer de endometrio (sin evidencias de enfermedad extrauterina, excepto citología positiva) corresponde al 12 a 14\% de los casos.

2. Diversos autores han reportado deterioro del pronóstico al tener citologías positivas para células neoplásicas en la cavidad peritoneal. Los estudios mas relevantes al respecto son: 1) El GOG 33 (8) con 697 pacientes estadio I operadas con etapificación quirúrgica completa; la tasa de recurrencias fue de $29 \%$ para las portadoras de citología positiva vs. $10 \%$ cuando la citología resultó negativa. 2) La revisión de Milosevic y cols (9) con 17 estudios agrupando 3.820 pacientes etapa I tratados quirúrgicamente. Coincide en que la citología positiva tuvo peor pronóstico, pero lo asocia a factores intratumorales con mayor riesgo de recidiva.

3. Hasta el momento no existe evidencia suficiente para afirmar que tratar a estos pacientes en forma adyuvante sea mejor que no tratarlos. Posibles terapias adyuvantes son: radioterapia de abdomen total, P32 y manipulación con progestágenos.

4. Si efectivamente la manipulación y/o insuflación uterina aumentan la presencia de células tumorales en el peritoneo, estas prácticas debieran ser evitadas, a pesar que no se sabe realmente, si esto constituye per se un factor de mal pronóstico.

5. Se ignora si estos estadios IIIA inducidos 
por el procedimiento se comportan igual que los IIIA espontáneos.

Desde las declaraciones de la SGO, múltiples series retrospectivas (10-17) han reportado la presencia o ausencia de citología peritoneal positiva en pacientes con cáncer endometrial sometidos a histeroscopia u otras manipulaciones uterinas.

Biewenga y cols (17) son los únicos autores que reportan seguimiento a cinco años en pacientes con estas características: a 50 pacientes consecutivas con cáncer endometrial etapa I, se les realizó una histeroscopia, 33 días en promedio antes de la histerectomía, encontrándose $0 \%$ de citología peritoneal positiva para células neoplásicas. La sobrevida libre de enfermedad a cinco años fue $85 \%$ similar a los datos históricos locales.

Revel y cols, en una revisión no sistemática (18) concluye que no existen estudios randomizados sobre el tema y sólo datos retrospectivos muestran correlación entre histeroscopia con medios de distensión líquida y citología peritoneal positiva. Según este autor, el procedimiento estaría asociado a un mayor riesgo de contaminación peritoneal, pero no es posible determinar una relación de causalidad, ni establecer el riesgo oncológico de la histeroscopia, ni afirmar que esta empeore el pronóstico. En esta revisión son destacables algunas observaciones:

1. Los porcentajes de citologías (+) post-histeroscopía en pacientes con patología benigna varían según el medio de distensión utilizado: líquido de alta viscosidad (dextran) 42 a $100 \%$ de los casos; de baja viscosidad (Ringer o solución fisiológica), 25 a $57 \%$; y con $\mathrm{CO}_{2}, 0$ a $25 \%$ de los casos.

2. Con presiones de distensión de la cavidad uterina inferiores a $70 \mathrm{mmHg}$ no habría diseminación transtubaria.

3. La contaminación peritoneal sería mayor en la fase proliferativa del endometrio.

4. Se ha demostrado viabilidad en cultivo celular del material endometrial recolectado en el peritoneo de pacientes operadas de cáncer de endometrio.

5. La citología (+) postlegrado vs posthisteroscopia en perimenopáusicas con sangrado uterino anormal (SUA) no serían muy diferentes. Tampoco en cáncer de endometrio frente a diversas manipulaciones uterinas (14) (post-BEM 9\%, postlegrado $10 \%$ y posthisteroscopia $13 \%$ ).

En relación a esto último, Wang y Guo (19) reportan un estudio comparativo de 156 pacientes con cáncer endometrial sometidas a legrado vs. histeroscopia informando el hallazgo de citología peritoneal (+) en $32 \%$ vs. $35 \%$, respectivamente.

La única revisión sistemática de la literatura es la de Yazbeck y cols (20), quien encuentra cinco estudios adecuados para un metaanálisis: éste incluye 756 pacientes con cáncer endometrial de las cuales $40 \%$ fueron sometidas a histeroscopia preoperatoria y $60 \%$ fueron a legrado biópsico como estudio preoperatorio. El $10 \%$ de todas las pacientes presentaron citología peritoneal positiva para células neoplásicas, sin diferencia significativa entre las que tenían el antecedente de una histeroscopia previa. Las conclusiones de esta revisión son:

1. La histeroscopia diagnóstica no aumenta la frecuencia de células neoplásicas en el peritoneo

2. No hay respaldo para plantear un peor pronóstico en las pacientes con citología positiva secundaria a la histeroscopia

3. No existe razón para evitar la histeroscopia diagnóstica en el estudio inicial del cáncer endometrial.

A pesar de todo ello, desde el año 2000 no ha habido nuevas declaraciones de la SGO sobre el tema, manteniéndose hasta hoy vigentes sus recomendaciones. Una encuesta reciente (21) a 450 miembros de esta sociedad concluye lo siguiente: El $65 \%$ de los encuestados piensa que no debería removerse la citología peritoneal de la etapificación FIGO actualmente vigente. El $86 \%$ indicó que su decisión en términos de indicar tratamiento adyuvante se basa en estos casos en los factores de riesgo intratumorales para recidiva (grado de diferenciación, histología, penetración tumoral, etc.) y que el hecho de que la paciente hubiera sido sometida a una histeroscopia previa no modifica su decisión.

Es interesante examinar la experiencia japonesa publicada por Tanizawa y cols (22) en 1991 que reporta datos de 3681 pacientes con cáncer de endometrio con diferentes estadios tratados en 167 hospitales. De ellas, 1040 tenían el antecedente de una histeroscopia preoperatoria además del curetaje fraccionado de etapificación. Las pacientes no fueron randomizadas. La citología, evaluada al momento de la laparotomía no fue diferente entre el grupo con y sin histeroscopia. El autor concluye que la histeroscopia puede ser de valor en el estudio preoperatorio del cáncer endometrial y que no existe efecto apreciable en la citología peritoneal.

A la luz de la evidencia existente en la literatura, dada la falta de rigor metodológico de las publicaciones, no podemos concluir que la histeros- 
copia sea inocua en términos del riesgo de diseminación de células neoplásicas. Evidentemente tampoco se puede aseverar lo contrario, por lo que, seguirá siendo aconsejable evitar la insuflación y manipulación uterina excesiva en casos sospechosos o confirmados, para eliminar o disminuir la contaminación peritoneal.

En la práctica clínica el cáncer endometrial es diagnosticado principalmente a través de BEM (23). Las ventajas de este procedimiento ambulatorio son su buen rendimiento diagnostico y su validación como la estrategia de menor costo beneficio (24) cuando la prevalencia de cáncer endometrial es mayor al $15 \%$ y cuando la metrorragia en mujeres postmenopáusicas se asocia a endometrio engrosado en EcoTv.

\section{RENDIMIENTO DIAGNÓSTICO DE LA HISTEROSCOPIA}

El diseño metodológico que mejor evalúa un procedimiento diagnóstico es el de corte transversal. Sin embargo, en la literatura médica existen más de 3000 artículos relacionados con la histeroscopia, la mayoría de ellos no cumplen con esta característica por lo que la forma de abordar la data existente es a través de revisiones sistemáticas y metaanálisis:

En la Tabla I se condensan los resultados de 43.757 pacientes estudiadas en 7 metaanálisis (4,25-30) que evalúan diversos procedimientos diagnósticos, utilizando como control la histopatología. La limitante de estos estudios corresponde a las características de la población estudiada (diversas proporciones de mujeres premenopáusicas y postmenopáusicas, sintomáticas y no sintomáticas), como también del origen de la muestra analizada (histerectomía, legrado, biopsia dirigida). A pesar de los sesgos enunciados, es posible concluir lo siguiente:

1. La EcoTv es un examen bien tolerado por las pacientes, con mínimo porcentaje de falla a diferencia de la $\mathrm{Hx}$, BEM e HSG, procedimientos que tienen un porcentaje de fracaso de 4 a $10 \%$.

2. Los exámenes ultrasonográficos (EcoTv e HSG) poseen alta sensibilidad (92-96\%) pero mala especificidad (61-88\%). En contraste, la BEM y la $\mathrm{Hx}$ presentan una sensibilidad modesta $(75-86 \%)$ y una muy alta especificidad (96-99\%). Ello los hace complementarios en clínica: estudio primario con EcoTv y definición diagnóstica con BEM o Hx.

3. La EcoTv frecuentemente se equivoca en predecir cáncer y patología endometrial. Es mejor examen para descartarlos, aunque presenta un $8 \%$ de falsos negativos para cáncer endometrial. Por ello se sugiere continuar estudio en síntomas persistentes o concentración de factores de riesgo. En postmenopáusicas el umbral de $5 \mathrm{~mm}$ determina qué pacientes deben continuar con estudios histológicos

4. Tanto Hx como BEM presentan mayor capacidad diagnóstica para cáncer en comparación con patología endometrial.

5. En los estudios de histeroscopia, la prevalencia de lesiones focales en la cavidad uterina fue $18 \%$ y tanto su sensibilidad como su especificidad para cáncer endometrial se mantuvieron en presencia de aquellas.

6. Los estudios de HSG no evalúan su rendimiento en cáncer endometrial. HSG mejora la ca-

Tabla I

COMPARACIÓN DEL RENDIMIENTO DIAGNÓSTICO DE EXÁMENES PARA EL ESTUDIO ENDOMETRIAL. COMPILACIÓN DE 7 METAANÁLISIS QUE RESUMEN EVALUACIONES EN 43.757 PACIENTES

\begin{tabular}{|c|c|c|c|c|c|c|c|}
\hline Examen & \multicolumn{2}{|c|}{ Histeroscopia (25) } & \multicolumn{2}{|c|}{ Bx endometrial (26-28) } & \multicolumn{2}{|c|}{ Eco TV $(4,29)$} & HSG (30) \\
\hline Estudios (n) & \multicolumn{2}{|c|}{65} & \multicolumn{2}{|c|}{39} & \multicolumn{2}{|c|}{35} & 24 \\
\hline Pacientes (n) & \multicolumn{2}{|c|}{26.346} & \multicolumn{2}{|c|}{9808} & \multicolumn{2}{|c|}{5.892} & 1.711 \\
\hline \multirow[t]{2}{*}{ Tasa de fallos } & \multicolumn{2}{|c|}{$4 \%$} & \multicolumn{2}{|c|}{$10 \%$} & \multicolumn{2}{|c|}{$0 \%$} & $7 \%$ \\
\hline & $\begin{array}{l}\text { Ca. endo- } \\
\text { metrio }\end{array}$ & $\begin{array}{c}\text { Patología } \\
\text { endometrial }\end{array}$ & $\begin{array}{l}\text { Ca. endo- } \\
\text { metrio }\end{array}$ & $\begin{array}{c}\text { Patología } \\
\text { endometrial }\end{array}$ & $\begin{array}{c}\text { Ca. endo- } \\
\text { metrio }\end{array}$ & $\begin{array}{c}\text { Patología } \\
\text { endometrial }\end{array}$ & $\begin{array}{c}\text { Patología } \\
\text { endometrial }\end{array}$ \\
\hline Sensibilidad & $86 \%$ & $78 \%$ & $68-81 \%$ & $75 \%$ & $96 \%$ & $92 \%$ & $95 \%$ \\
\hline $\begin{array}{l}\text { Especificidad } \\
\text { PPT }\end{array}$ & $99 \%$ & $96 \%$ & $99 \%$ & $99 \%$ & $61 \%$ & $81 \%$ & $88 \%$ \\
\hline Positivo & $72 \%$ & $55 \%$ & $82 \%$ & $58 \%$ & $31 \%$ & $31-57 \%$ & $91 \%$ \\
\hline Negativo & $0,6 \%$ & $2,8 \%$ & $0,9 \%$ & $2 \%$ & $2,5 \%$ & $1 \%$ & $7 \%$ \\
\hline
\end{tabular}

Bx: biopsia. Eco TV: ecografía transvaginal. HSG: histerosonografía. Ca: cáncer. PPT: probabilidad post test. ( ): referencia bibliográfica. 
racterización de lesiones focales visibles o sospechadas en EcoTv.

Clark (31) en una revisión sistemática de la literatura concuerda con lo señalado y agrega lo siguiente respecto a $\mathrm{Hx}$ ambulatoria, EcoTv e HSG:

1. Hay ventajas específicas para cada examen: EcoTv e HSG permiten diagnóstico de eventuales patologías extra cavitarias con la adición de doppler y 3D es posible que mejoren su precisión diagnóstica. Hx tiene capacidad no solo diagnóstica sino también terapéutica.

2. El rendimiento diagnóstico de BEM e $\mathrm{Hx}$ para cáncer e hiperplasia endometrial es alto tanto en pre como en postmenopáusicas.

3. En postmenopáusicas sintomáticas, $\mathrm{Hx}(+)$ es superior a EcoTv para predecir patología endometrial; mientras que EcoTv (-) es más segura para descartarla.

4. En premenopáusicas sintomáticas EcoTv no tiene umbral de corte específico para diagnóstico de patología endometrial.

5. En relación a pólipos endometriales, HSG es más sensible y específica que EcoTv para su diagnóstico. Por otra parte, la probabilidad de encontrar cáncer o patología endometrial en un pólipo se estima en 1 y $20-30 \%$ respectivamente.

\section{CONCLUSIONES}

De acuerdo a los antecedentes examinados, y mientras no exista una demostración concluyente de la inocuidad de la histeroscopia en cáncer endometrial, parece prudente abstenerse de realizar histeroscopias en casos sospechosos. Siempre que sea posible, será preferible hacer el diagnóstico con un dispositivo que obtenga tejido endometrial y que contamine lo menos posible la cavidad peritoneal con células neoplásicas. Sin embargo, dado que el riesgo de la contaminación es probablemente bajo, la $\mathrm{Hx}$ y la biopsia dirigida tienen indicación toda vez que los otros procedimientos no permitan la certeza diagnostica.

Si bien existen múltiples publicaciones en relación al estudio de patología endometrial y los riesgos asociados a los procedimientos diagnósticos y/o terapéuticos empleados, no existe aun un protocolo de estudio suficientemente validado. De acuerdo a lo examinado y valorando los estudios randomizados controlados y publicados últimamente $(3,32)$, existirían esfuerzos para lograrlo. Sin embargo, a falta de protocolos definidos, existen algunas recomendaciones y conceptos generales que pueden aplicarse al estudio del endometrio sintomático:
1. La EcoTv en el estudio inicial de la metrorragia es un examen de fácil aplicación, sensible y de bajo costo.

2. Todos los procedimientos ambulatorios invasivos de la cavidad uterina, tienen una tolerancia y posibilidad de ser completados más o menos similar. La histeroscopia ambulatoria no es superior a la BEM ni a la HSG. Distinto es el caso de la histeroscopia bajo anestesia, la cual puede compararse con el legrado uterino formal, donde ambos procedimientos requieren de dilatación del cuello uterino.

3. En la metrorragia post menopáusica con endometrio grueso diagnosticado por EcoTv, la BEM es la estrategia más económica y simple para descartar cáncer endometrial con un aceptable nivel de certeza.

4. En la metrorragia post menopáusica con endometrio fino diagnosticado por EcoTv, si el síntoma es aislado, se sugiere considerar el diagnóstico atrofia endometrial y controlar clínicamente a la paciente. $\mathrm{Si}$ el sangrado persiste o la paciente concentra factores de riesgo para cáncer endometrial la BEM también está indicada. Sin embargo, si ésta es impracticable, su resultado es no concluyente por muestra escasa u otra razón, la histeroscopia y biopsia dirigida o seguida de legrado sistemático de la cavidad son los procedimientos más seguros para descartar cáncer endometrial. Recordar siempre que la sensibilidad de histeroscopia se mantiene aún en presencia lesiones focales.

5. En mujeres pre y postmenopáusicas con metrorragia cuya EcoTv sugiere lesiones focales, la histeroscopia tiene una capacidad diagnostica superior al resto de los exámenes evaluados en esta revisión, seguida luego de la histerosonografía. La histeroscopia permite biopsia dirigida inmediata y resolución quirúrgica en el mismo acto incluso en forma ambulatoria.

Por otra parte, en mujeres premenopáusicas con metrorragia y endometrio normal en EcoTv (homogéneo y de un grosor inferior a 16 milímetros), lo primero a considerar son los trastornos disfuncionales. Sin embargo, si el endometrio se mantiene engrosado, los síntomas persisten o resultan refractarios a los tratamientos hormonales, se hace necesario descartar patología endometrial. Nuevamente, la BEM representa una alternativa barata y útil, aunque debemos recordar que en esta situación clínica será menos sensible que una histeroscopia. Lo anterior debido a que en esta edad es menos probable encontrar un cáncer de endometrio. Parece entonces aceptable la 
histeroscopia sin estudio histológico previo cuando la sospecha de cáncer no es francamente alta.

\section{BIBLIOGRAFÍA}

1. Gimpelson RJ, Rappold HO. A comparative study between panoramic hysteroscopy with directed biopsies and dilatation and curettage. Am J Obstet Gynecol 1988;158:489-92.

2. Clark TJ, Khan KS, Gupta JK. The diagnosis of intrauterine pathology in postmenopausal women: an evidence-based approach. Rev Gynaecol Pract 2002;2:109-16.

3. Critchley HO, Warner P, Lee AJ, Brechin S, Guise J, Graham B. Evaluation of abnormal uterine bleeding: comparison of three outpatient procedures within cohorts defined by age and menopausal status. Health Technol Assess. 2004;8(34):1-139.

4. Smith-Bindman R, Kerlikowske $\mathrm{K}$, Feldstein VA, Subak L, Scheidler J, Segal M, Brand R, Grady D. Endovaginal ultrasound to exclude endometrial Cancer and other endometrial abnormalities. JAMA 1998;280(17):1510-7.

5. Herzog TJ. Peritoneal Cytology Issues in Endometrial Cancers: Possible latrogenic Positive Washings. www.medscape.com/viewarticle/413007, Medscape coverage of 31 st Annual Meeting of the SGO, February 5-9, 2000; San Diego, California.

6. Zerbe MJ, Zhang J, Bristow RE, Grumbine FC, Abularach SM, Montz FJ. Retrograde seeding of malignant cells during hysteroscopy in endometrial cancer. Program and abstracts of the 31st Annual Meeting of the Society of Gynecologic Oncologists; February 5-9, 2000; San Diego, California. Abstract 22.

7. Sonoda Y, Zerbe M, Barakat RR, et al. High incidence of positive peritoneal cytology in low-risk endometrial cancer treated by laparoscopically assisted vaginal hysterectomy (LAVH). Program and abstracts of the 31st Annual Meeting of the Society of Gynecologic Oncologists; February 5-9, 2000; San Diego, California. Abstract 21.

8. Morrow CP, Bundy BN, Kurman RJ, et al. Relationship between surgical-pathologic risk factors and outcome in clinical stage I and II carcinoma of the endometrium: a Gynecologic Oncology Group study. Gynecol Oncol 1991;40:55.

9. Milosevic MF, Dembo AJ, Thomas GM. The clinical significance of malignant peritoneal cytology in stage I endometrial carcinoma. Int J Gynecol Cancer 1992;2:225.

10. Obermair A, Geramou M, Gucer F, Denison U, Graf AH, Kapshammer E, Neunteufel W, Frech I, Kaider A, Kainz C. Does hysteroscopy facilitate tumor cell dissemination? Incidence of peritoneal cytology from patients with early stage endometrial carcinoma following dilatation and curettage $(D \& C)$ vs. hysteroscopy and D \& C. Cancer 2000;88(1):139-43.

11. Lo KW, Cheung TH, Yim SF, Chung TK. Hysteros- copic dissemination of endometrial carcinoma using carbon dioxide and normal saline: a retrospective study. Gynecol Oncol. 2002;84(3):394-8.

12. Kudela M, Pilka R. Is there a real risk in patients with endometrial carcinoma undergoing diagnostic hysteroscopy (HSC)? Eur J Gynaecol Oncol 2001; 22(5):342-4.

13. Selvaggi L, Cormio G, Ceci O, Loverro G, Cazzolla A, Bettocchi S. Hysteroscopy does not increase the risk of microscopic extrauterine spread in endometrial carcinoma. Int J Gynecol Cancer 2003;13(2): 223-7.

14. Lo KW, Cheung TH, Yim SF, Yu MY, Chan LY, Chung TK. Prospective self-controlled study on prevention of hysteroscopic dissemination in endometrial carcinoma. Int J Gynecol Cancer. 2004;14(5): 921-6.

15. Gu M, Shi W, Huang J, Barakat RR, Thaler HT, Saigo PE. Association between initial diagnostic procedure and hysteroscopy and abnormal peritoneal washings in patients with endometrial carcinoma. Cancer 2000;90(3):143-7.

16. Obermair A, Geramou M, Gucer F, Denison U, Graf $\mathrm{AH}$, Kapshammer E, Medl M, Rosen A, Wierrani F, Neunteufel W, Frech I, Preyer O, Speiser P, Kainz C. Impact of hysteroscopy on disease-free survival in clinically stage I endometrial Cancer patients. Int J Gynecol Cancer 2000;10(4):275-279.

17. Biewenga P, de Blok S, Birnie E. Does diagnostic hysteroscopy in patients with stage I endometrial carcinoma cause positive peritoneal washings? Gynecol Oncol 2004;93(1):194-8.

18. Revel A, Tsafrir A, Anteby SO, Shushan A. Does hysteroscopy produce intraperitoneal spread of endometrial Cancer cells? Obstet Gynecol Surv 2004; 59(4):280-4.

19. Wang W, Guo Y. Value of hysteroscopy and dilatation and curettage in diagnosis of endometrial carcinoma. Zhonghua Fu Chan Ke Za Zhi 2002;37(9): 550-2.

20. Yazbeck C, Dhainaut C, Batallan A, Benifla JL, Thoury A, Madelenat P. Diagnostic hysteroscopy and risk of peritoneal dissemination of tumor cells Gynecol Obstet Fertil. 2005;33(4): 247-52. Epub 2005.

21. Lee CM, Slomovitz BM, Greer M, Sharma S, Gregurich MA, Burke T, Lu KH, Ramondetta LM. Practice patterns of SGO members for stage IIIA endometrial Cancer. Gynecol Oncol 2005;98(1):7783.

22. Tanizawa O, Miyake A, Sugimoto O. Re-evaluation of hysteroscopy in the diagnosis of uterine endometrial Cancer. Nippon Sanka Fujinka Gakkai Zasshi 1991;43(6):622-6.

23. Amant F, Moerman P, Neven P, Timmerman D, Van Limbergen E, Vergote I. Endometrial Cancer. Lancet 2005;366(9484):491-505.

24. Dijkhuizen FP. Cost-effectiveness of the use of transvaginal sonography in the evaluation of postmenopausal bleeding. Maturitas 2003;45(4):275-82. 
25. Clark TJ, Voit D, Gupta JK, Hyde C, Song F, Khan KS. Accuracy of hysteroscopy in the diagnosis of endometrial Cancer and hyperplasia: a systematic quantitative review. JAMA 2002;288(13):1610-21.

26. Dijkhuizen FP, Mol BW, Brolmann HA, Heintz AP. The accuracy of endometrial sampling in the diagnosis of patients with endometrial carcinoma and hyperplasia: a meta-analysis. Cancer 2000;89(8): 1765-72.

27. Clark TJ, Mann CH, Shah N, Khan KS, Song F, Gupta JK. Accuracy of outpatient endometrial biopsy in the diagnosis of endometrial Cancer: a systematic quantitative review. BJOG 2002;109(3):313-21.

28. Clark TJ, Mann CH, Shah N, Khan KS, Song F, Gupta JK. Accuracy of outpatient endometrial biopsy in the diagnosis of endometrial hyperplasia. Acta Obstet Gynecol Scand 2001;80(9):784-93.
29. Gupta JK, Chien PF, Voit D, Clark TJ, Khan KS. Ultrasonographic endometrial thickness for diagnosing endometrial pathology in women with postmenopausal bleeding: a meta-analysis. Acta Obstet Gynecol Scand 2002;81(9):799-816.

30. de Kroon CD, de Bock GH, Dieben SW, Jansen FW. Saline contrast hysterosonography in abnormal uterine bleeding: a systematic review and meta-analysis. BJOG 2003;110(10):938-47.

31. Clark TJ. Outpatient hysteroscopy and ultrasonography in the management of endometrial disease. Curr Opin Obstet Gynecol 2004;16:305-11.

32. Bain C, Parkin DE, Cooper KG. Is outpatient diagnostic hysteroscopy more useful than endometrial biopsy alone for the investigation of abnormal uterine bleeding in unselected premenopausal women? A randomized comparison. BJOG 2002;109(7):805-11. 